\title{
Dynamics of nonequilibrium quasiparticles in narrow-gap superconducting tunnel junctions
}

\author{
A. G. Kozorezov, ${ }^{1}$ R. A. Hijmering, ${ }^{2}$ G. Brammertz, ${ }^{2}$ J. K. Wigmore, ${ }^{1}$ A. Peacock, ${ }^{2}$ D. Martin, ${ }^{2}$ P. Verhoeve, ${ }^{2}$ \\ A. A. Golubov, ${ }^{3}$ and H. Rogalla ${ }^{3}$ \\ ${ }^{1}$ Department of Physics, Lancaster University, Lancaster LA1 4YB, United Kingdom \\ ${ }^{2}$ Science Payloads and Advanced Concepts Office, SCI-A, ESTEC, European Space Agency, Noordwijk, The Netherlands \\ ${ }^{3}$ Department of Applied Physics, University of Twente, P.O. Box 217, 7500 AE Enschede, The Netherlands
}

(Received 17 July 2007; revised manuscript received 24 September 2007; published 2 January 2008)

\begin{abstract}
The latest generation of high quality, narrow gap, superconducting tunnel junctions (STJs) exhibits a steadystate and time-dependent behavior which cannot be described satisfactorily by previous treatments of nonequilibrium quasiparticle (qp) dynamics. These effects are particularly evident in experiments using STJs as detectors of photons, over the range from near infrared to $\mathrm{x}$ ray. In this paper, we present a detailed theoretical analysis of the spectral and temporal evolution of the nonequilibrium qp and phonon distributions in such STJs excited by single photons, over a wide range of excitation energy, bias voltage, and temperature. By solving the coupled set of kinetic equations describing the interacting excitations, we show that the nonequilibrium qp distribution created by the initial photoabsorption does not decay directly back to the initial undisturbed state in thermal equilibrium. Instead, it undergoes a rapid adiabatic relaxation to a long-lived, excited state, the spectral distribution of which is nonthermal, maintained by a balance between qp creation, recombination, and trapping. The model is able to describe successfully photoabsorption data taken on several different aluminum STJs, using a single set of parameters. Of particular note is the conclusion that the local traps responsible for qp loss are situated specifically in the region of $\mathrm{Nb}$ contacts.
\end{abstract}

DOI: 10.1103/PhysRevB.77.014501

PACS number(s): 74.25.Fy

\section{INTRODUCTION}

The phenomenon of tunneling has been widely used as a tool to explore the basic physics of superconductivity, as well as to provide the underlying principle for numerous superconducting devices. ${ }^{1}$ There is currently great interest in using high quality superconducting tunnel junctions as single photon detectors for astrophysical and other applications. ${ }^{2}$ Such detectors operate under highly nonequilibrium conditions which do not occur in any other experimental scenario. Their performance is very sensitive to microscopic details of the quasiparticle (qp) dynamics and hence provides a unique opportunity for studying nonequilibrium behavior of the $q p$ population.

As a photon detector, the superconducting tunnel junction (STJ) possesses excellent responsivity (charge output per unit photon energy input), energy resolution, and count rate capability over a broad spectral range, from near infrared to hard $x$ ray. ${ }^{3-6}$ In order to optimize these characteristics, recent work at ESA and elsewhere has been focused on the development of improved devices having smaller energy gaps, highly homogeneous and transparent barriers, and extremely low quasiparticle loss rates. A key parameter is the energy gap, and first generation $\mathrm{Nb}$ STJs were succeeded by $\mathrm{Ta}$, then by proximized $\mathrm{Nb} / \mathrm{Al}$ and $\mathrm{Ta} / \mathrm{Al}$ ones, and most recently by pure Al structures, with energy gap one-eighth that of $\mathrm{Nb}$. While, as expected, the new devices exhibit significantly improved detector characteristics, several totally new phenomena have also emerged, including internal amplification due to qp back tunneling, ${ }^{7}$ enhanced tunneling and phonon noise, ${ }^{8,9}$ and time-dependent tunneling statistics. ${ }^{10,11}$ However, the most exciting discovery has been that of a whole new class of phenomena related to the formation of a nonequilibrium, coupled qp-phonon state due to multiple tunneling under bias.
The observation of this nonequilibrium situation in steady-state experiments and the explanation of its origins have been reported earlier. ${ }^{12,13}$ In a low loss, narrow band gap, multitunneling device, qps may retain accumulated energy of $2 e V$, where $V$ is the bias voltage, for each cycle of forward and back tunneling before relaxation. In this context, a narrow gap STJ is the one in which a qp may tunnel several times before relaxing to a lower energy state. This should not be confused with multiple tunneling, when a qp tunnels several times with or without relaxation, before recombination takes place. ${ }^{13}$ As a consequence, a stable nonequilibrium state is established in which many qps may have energy exceeding the $3 \Delta$ threshold for breaking further Cooper pairs, resulting in an additional excess current and an increase in generation-recombination noise. ${ }^{14,15}$ The development of a quantitative theory of the phenomenon required the solution of the coupled system of kinetic equations for the interacting qps and phonons, resulting in a set of spectral balance equations describing the qp populations in different energy intervals.

The objective of the present work has been to carry out parallel calculations for the dynamic situation created by the absorption of a single photon in the energy range between near infrared and $\mathrm{x}$ ray. The time-dependent scenario is significantly more complicated than the stationary one, and a full solution including spectral balance within the qp and phonon distributions has never been achieved previously. Until recently, the only attempts to model the response of a biased STJ to the absorption of a photon creating a population of nonequilibrium qps used the framework of the Rothwarf-Taylor (RT) balance equations. ${ }^{16}$ The main assumption of this approach is that during the initial downconversion process, qps relax directly to states at the superconducting edge. However, for the latest STJs, the modeled results for charge output and its rise time as functions of bias 
voltage and temperature, both of which influence qp distributions, do not agree with the experimental data. Following more realistic calculations based on balancing the two processes of tunneling and spontaneous phonon emission, ${ }^{17,18}$ it is clear that the RT approach is too simplistic to treat satisfactorily a nonequilibrium situation. An attempt to tackle the problem was made recently by Segall et al. ${ }^{19}$ beginning from a phenomenologically derived system of rate equations to describe the dynamical situation. However, this formulation did not include the complete kinetic equations for interacting qps and phonons and took no account of nonequilibrium phonon distributions or qp generation effects.

In order to be able to include nonequilibrium phenomena explicitly, we have developed a theoretical approach based on the projection of the exact kinetic equations for the qps and phonons on to a discretized energy space. Interacting distributions of qps and phonons are described in terms of a system of spectral balance equations with all scattering and interaction terms rigorously derived from the corresponding collision integrals. We previously used this approach to model successfully the nonequilibrium qp dynamics for a BCS superconductor in the stationary regime ${ }^{12,13}$ and for the general situation of a proximized structure with timeevolving distributions. ${ }^{20,21}$ However, the latter scheme was incomplete since it did not take account of the qp selfgeneration and used an oversimplified model of qp detrapping at localized traps. In addition, the effects of the nonequilibrium subgap phonon distribution were not considered. All effects are included in the present work.

In this paper, we develop a general technique for modeling nonequilibrium, time-dependent phenomena in superconducting tunnel junctions. For comparison, we also present recent experimental results taken on several narrow gap STJs of different sizes over a range of photon energy, bias voltage, and temperature. Convincing agreement between experimental results and theoretical predictions is obtained for all devices using a single set of fitting parameters.

The paper is organized as follows. In Sec. II, the various processes involved in the interactions between nonequilibrium and trapped qps and phonons are described. The resultant time-dependent spectral balance equations are derived in Sec. III. Section IV contains details of the experiments and modeling, followed by a comparison of experimental data with theoretical calculations in Sec. V, and a brief summary of our conclusions in Sec. VI.

\section{KINETIC DESCRIPTION OF NONEQUILIBRIUM QUASIPARTICLES AND PHONONS IN A SUPERCONDUCTING TUNNEL JUNCTION}

A fully dynamical description of nonequilibrium qps and phonons in an STJ begins from the kinetic equations for a superconductor with all qp and phonon processes including tunneling, represented by collision integrals. ${ }^{22,23}$ For qps and phonons, respectively, the equations are

$$
\frac{\partial f(\epsilon)}{\partial t}=I_{q p-p h}\{f, N\}+I_{\text {rec }}\{f\}+I_{\text {loss }}\{f\}+I_{\text {tun }}\{f, \tilde{f}\},
$$

$$
\frac{\partial N(\epsilon)}{\partial t}=I_{p h, l o s s}\{N\}+I_{p h-q p}\{N, f\}+I_{p b}\{N, f\} .
$$

Here, $f(\epsilon)$ and $N(\epsilon)$ are the respective distribution functions for qps and phonons, where $\epsilon$ is the qp energy relative to the Fermi level. Collision integrals in Eq. (1) describe the following qp processes: $I_{q p-p h}\{f, N\}$ relates to qp-phonon scattering processes with either emission or absorption of a single phonon, $I_{\text {rec }}\{f\}$ takes into account recombination, while $I_{\text {loss }}\{f\}$ incorporates processes other than recombination which also result in the loss of qps. The latter include trapping with subsequent recombination on the trapping site, and diffusion and loss in the lead connections. Finally, $I_{\text {tun }}\{f, \tilde{f}\}$ describes the rate of qp exchange with the other electrode, where the qp distribution function is described by $\widetilde{f}$. Collision integrals in the kinetic equation [Eq. (2)] for phonons are $I_{\text {ph,loss }}\{N\}$ taking account of phonon escape from the electrode, $I_{p h-q p}\{N, f\}$ relating to phonon reabsorption by qps, and $I_{p b}\{N, f\}$ describing the effect of Cooper pair breaking by phonons. The latter is nonzero only for energetic phonons with $\hbar \Omega>2 \Delta$, where $\Delta$ is the superconducting gap.

We showed earlier ${ }^{24,25}$ that the kinetics of qps and phonons in nonequilibrium superconductors cannot be adequately described without taking explicit account of the interaction between the mobile qps and phonons and the trapped qps. The microscopic nature of the defects responsible for the trapping states in a particular superconductor is often uncertain. Possible sources are magnetic impurities or clusters, macroscopic regions of locally suppressed gap such as the core regions of trapped magnetic flux, small normal metal inclusions, surface layers of smaller gap natural oxide and suppressed gap regions due to sample geometry. The role of these states in the nonequilibrium kinetics in superconductors can be compared with that of traps or deep levels in semiconductors. Their importance was first demonstrated in Ref. 24, and their effect can be seen in the dynamic response of the STJs to any transient perturbation. ${ }^{26}$ Although the production of qps in photon absorption experiments occurs in a tiny excited volume close to the photon absorption site, for a typical STJ only a few tens of microns in size, diffusion rapidly homogenizes their distribution over the whole electrode on a time scale which is much shorter than any of the processes which control subsequent evolution of the qp distribution. Thus, we may omit spatial gradients from the kinetic equations and, equally, we may disregard the positional dependence of qp trapping. We assume that trapping centers of depth $\Delta_{t}$ are distributed through the STJ with density $F_{t}$. Thus, the activation energy of the trapped qps is $\Delta-\Delta_{t}$.

As a consequence, the main system of equations must be modified to include additional terms representing the various interactions between the three subsystems, mobile qps, trapped qps, and phonons. Thus, instead of Eqs. (1) and (2), we may write

$$
\begin{aligned}
\frac{\partial f(\epsilon)}{\partial t}= & I_{q p-p h}\{f, N\}+I_{\text {rec }}\{f\}+I_{\text {rec }}\left\{f, f^{\text {trap }}\right\}+I_{\text {loss }}\{f\}+I_{\text {tun }}\{f, \tilde{f}\} \\
& +I_{\text {trap }}\left\{f, f^{\text {trap }}\right\}-I_{\text {detrap }}\left\{f^{\text {trap }}\right\},
\end{aligned}
$$




$$
\begin{aligned}
\frac{\partial f^{\text {trap }}}{\partial t}= & +I_{\text {loss }}\left\{f^{\text {trap }}\right\}-I_{\text {trap }}\left\{f, f^{\text {trap }}\right\}+I_{\text {detrap }}\left\{f, f^{\text {trap }}\right\} \\
& -I_{\text {rec }}\left\{f, f^{\text {trap }}\right\}-I_{p b}\left\{N, f, f^{\text {trap }}\right\}, \\
\frac{\partial N(\epsilon)}{\partial t}= & I_{p h, l o s s}\{N\}+I_{p h-q p}\{N, f\}+I_{p h-t r a p}\left\{N, f^{\text {trap }}\right\}+I_{p b}\{N, f\} \\
& +I_{p b}\left\{N, f, f^{\text {trap }}\right\} .
\end{aligned}
$$

Here, we introduce the notation $f^{\text {trap }}$ for the trapped qp density. The collision integrals $I_{\text {loss }}\left\{f^{\text {trap }}\right\}, I_{\text {trap }}\left\{f, f^{\text {trap }}\right\}$, and $I_{\text {detrap }}\left\{f, f^{\text {trap }}\right\}$ describe, respectively, the rate of qp loss due to recombination on the trap, the rate of qp trapping from mobile qp states, and the rate of trap depopulation. We have also split the recombination terms in Eq. (3) into two parts, the first $I_{\text {rec }}\{f\}$ accounting for the normal recombination of a test qp colliding with another mobile $\mathrm{qp}$ and the second $I_{\text {rec }}\left\{f, f^{t r a p}\right\}$ being the contribution due to the collision with a trapped qp. Similarly, in Eq. (3), we have split the phonon pair breaking term into the two terms with $I_{p b}\{N, f\}$ describing the process resulting in the creation of two mobile qps, while $I_{p b}\left\{N, f, f^{t r a p}\right\}$ leads to the creation of one trapped and one mobile qp. In what follows, we will assume that the number of traps is small, so that we may disregard $I_{\text {rec }}\left\{f, f^{\text {trap }}\right\}$ in comparison with $I_{\text {rec }}\{f\}$ in Eq. (3) and $I_{p b}\left\{N, f, f^{t r a p}\right\}$ in comparison with $I_{p b}\{N, f\}$ in Eq. (5). Similarly, in Eq. (4) we will disregard the terms with $I_{r e c}\left\{f, f^{t r a p}\right\}$ and $I_{p b}\left\{N, f, f^{t r a p}\right\}$, which describe the population of traps in the processes of recombination and pair breaking and are small in comparison with $I_{\text {trap }}\left\{f, f^{\text {trap }}\right\}$. However, quadratic terms of the kind $f f^{\text {trap }}$ must be retained in $I_{\text {detrap }}\left\{f, f^{\text {trap }}\right\}$ since detrapping may occur either through collision with a thermal phonon, with the strength of the process depending exponentially on temperature, or through depopulation of the trap by one of the nonequilibrium carriers, which may become important at low temperature and high nonequilibrium qp density.

The equation for the phonon distribution function [Eq. (5)] is a first order linear differential equation and can be solved in terms of the qp distribution function. It has been shown previously that, after the fast, initial energy down conversion, all succeeding evolution of the nonequilibrium qp distribution is controlled purely by tunneling loss and recombination, which occur much more slowly. Thus, the qp distribution in the biased STJ remains "frozen-in" and the energetic phonon distribution quickly accommodates itself to the slowly varying qp distribution. In this situation, for pairbreaking phonons with energy above $2 \Delta$, all processes in the phonon system occur much faster than those which control the nonequilibrium qp distribution. Hence, we can use an adiabatic approximation and neglect all effects of temporal dispersion of phonon response. By setting the time derivative of the phonon distribution to zero, we reduce the differential equation to an algebraic one, resulting in a coupled system of equations for mobile and trapped qps. This approach is not valid for lower energy subgap phonons as their loss rate may be very slow and the temporal response may become dispersive. ${ }^{13,27,28}$ In addition, their significant accumulation changes the rates of detrapping through the term $I_{\text {detrap }}\left\{f^{\text {trap }}\right\}$. However, for the moment, we will ignore this group of phonons but will discuss their possible role in the later consideration of real structures and experimental situations.

\section{TIME-DEPENDENT SPECTRAL BALANCE EQUATIONS}

Time-dependent spectral balance equations were previously derived in Refs. 20 and 21 for the general case of a proximized STJ. However, nonequilibrium phonon effects were only partially taken into account, through simplified phonon reabsorption terms in the collision integrals describing the recombination. Conversely, in Ref. 13, the phonon contribution was fully accounted for, but only for the stationary situation. In the previous work, we used the expressions for the collision integrals in Eqs. (3)-(5) and projecting these kinetic equations onto energy space as has been described in Refs. 13 and 20, we obtained a system of coupled spectral balance equations for qps. The energy range of interest is split into $M+1(M \gg 1)$ elementary intervals with width $\delta$, labeled by the integer $m$, so that the $m$ th elementary interval in energy space becomes $\epsilon_{m}<\epsilon<\epsilon_{m+1}$, where $\epsilon_{m}=\Delta+m \delta$ and the index $m$ defines the qp energy relative to the gap, $\Delta$. The number $M$ is chosen so that $M \delta \geqslant 3 \Delta$ falls into the active region defined as $\epsilon \geqslant 3 \Delta$, so that the inelastic relaxation of a qp from this region may release a pair-breaking phonon, leading to qp generation. ${ }^{13}$ We take only values of bias voltage which are integer numbers of the elementary width $\delta$, that is, $V_{b}=v \delta$. The trap depth measured from the superconducting edge is also assumed to be an integer multiple of $\delta$, so that $\Delta-\Delta_{t}=t \delta$.

After the transformation of Eqs. (3) and (4), our main equations for mobile [Eq. (6)] and trapped [Eq. (7)] qps become

$$
\begin{aligned}
\frac{\partial P_{m}^{i}}{\partial t}= & -\frac{P_{m}^{i}}{\tau_{m}}+\sum_{s=m+1}^{M} \frac{P_{s}^{i}}{\tau_{s \rightarrow m}}-\frac{P_{m}^{i}-P_{m}^{0}}{\tau_{l, m}}-\Gamma_{m \rightarrow m+v} P_{m}^{i} \\
& +\Gamma_{m+v \rightarrow m} P_{m+v}^{j}-\Theta(m-v+0)\left[\Gamma_{m \rightarrow m-v} P_{m}^{i}\right. \\
& \left.-\Gamma_{m-v \rightarrow m} P_{m-v}^{j}\right]-2 \bar{N}(0) \Delta \sum_{s=0}^{M} R_{m, s}^{*}\left[P_{m}^{i} P_{s}^{i}-P_{m}^{0} P_{s}^{0}\right] \\
& -\frac{P_{m}^{i}}{\tau_{m \rightarrow \text { trap }}}\left(1-\frac{f^{t}}{F^{t}}\right)+\frac{f^{t}}{\tau_{\text {trap } \rightarrow m}} \\
& +f^{t} \sum_{s=m+t}^{M} \int_{0}^{t} \frac{d t^{\prime}}{\tau_{0}} P_{s}^{i}\left(t^{\prime}\right) K_{m, s}\left(t-t^{\prime}\right)+\sum_{s=m+c e i l(2 \Delta / \delta)}^{M} \frac{P_{s}^{i}}{\tau_{g, s}},
\end{aligned}
$$

$$
\begin{aligned}
\frac{\partial f^{t}}{\partial t}= & -\frac{f^{t}-f_{0}^{t}}{\tau_{\text {trap-loss }}}+\sum_{s=0}^{M} \frac{P_{s}^{i}}{\tau_{s \rightarrow \text { trap }}}\left(1-\frac{f^{t}}{F^{t}}\right)-\frac{f^{t}}{\tau_{\text {detrap }}} \\
& -f^{t} \sum_{s=0}^{M} \sum_{s^{\prime}=s+t}^{M} \int_{0}^{t} \frac{d t^{\prime}}{\tau_{0}} P_{s^{\prime}}^{i}\left(t^{\prime}\right) K_{s, s^{\prime}}\left(t-t^{\prime}\right) \\
& -2 \bar{N}(0) \Delta \sum_{s=c e i l\left(\Delta_{t} / \delta\right)}^{M} R_{\text {trap }, s}\left[P_{s}^{i} f^{t}-P_{s}^{0} f_{0}^{t}\right],
\end{aligned}
$$


where $\operatorname{ceil}(x)$ is the smallest integer greater than or equal to $x$. Here, we have introduced $P_{s}^{i}$, which is the dimensionless density in units of $2 \bar{N}(0) \Delta$ of qps which belong to the $s$ th interval, and $P_{s}^{0}$ is its value for thermally excited qps. The superscript $i$ labels the base and $j$ the top STJ electrodes, and $\bar{N}(0)$ is the density of states at the Fermi level in the normal state, per spin. Similarly, $f^{t}$ is the dimensionless density of trapped qps in the same units, $f_{0}^{t}$ is its equilibrium value, and $F^{t}$ is the overall dimensionless trap density including both occupied and vacant traps.

Other kinetic parameters in Eqs. (6) and (7) are defined as follows: ${ }^{13}$

$$
\frac{1}{\tau_{m}}=\frac{1}{\tau_{0}}\left(\frac{\Delta}{k_{B} T_{c}}\right)^{3} \int_{\Delta}^{\epsilon_{\max }} \frac{d \epsilon^{\prime}}{\Delta} \rho\left(\epsilon^{\prime}\right) \bar{B}\left(\epsilon^{\prime}, \epsilon_{m}\right),
$$

where $B\left(\epsilon, \epsilon^{\prime}\right)=\left[\left(\epsilon-\epsilon^{\prime}\right) / \Delta\right]^{2}\left(1-\Delta^{2} / \epsilon \epsilon^{\prime}\right) \Theta\left(\epsilon^{\prime}-\epsilon\right) \quad$ and $\Theta\left(\epsilon^{\prime}-\epsilon\right)$ is the Heaviside function, so that

$$
\bar{B}\left(\epsilon^{\prime}, \epsilon_{m}\right)=\frac{1}{\rho_{m}} \int_{\epsilon_{m}}^{\epsilon_{m+1}} d \epsilon \rho(\epsilon) B\left(\epsilon^{\prime}, \epsilon\right)
$$

is the average over the $m$ th spectral interval, where

$$
\rho_{m}=\int_{\epsilon_{m}}^{\epsilon_{m+1}} \frac{d \epsilon}{\Delta} \rho(\epsilon) .
$$

Here, $T_{c}$ is critical temperature, $\tau_{0}$ is the characteristic electron-phonon relaxation time in the superconductor, and $\rho(\epsilon)$ is the qp dimensionless density of states. It is seen from Eq. (8) that $\tau_{m}$ is the lifetime of a qp with respect to scattering from the initial state in the $m$ th interval down to any lower lying state with the spontaneous emission of a phonon. Similarly,

$$
\frac{1}{\tau_{s \rightarrow m}}=\frac{1}{\tau_{0}}\left(\frac{\Delta}{k_{B} T_{c}}\right)^{3} \int_{\epsilon_{m}}^{\epsilon_{m+1}} \frac{d \epsilon^{\prime}}{\Delta} \rho\left(\epsilon^{\prime}\right) \bar{B}\left(\epsilon^{\prime}, \epsilon_{s}\right),
$$

where $\tau_{s \rightarrow m}$ describes the rate of electronic transition of a qp with the spontaneous emission of a phonon from an initial state in the sth interval to any state in the $m$ th interval. The loss rate for qps belonging to the $m$ th interval is

$$
\frac{1}{\tau_{l, m}}=\frac{1}{\rho_{m}} \int_{\epsilon_{m}}^{\epsilon_{m+1}} d \epsilon^{\prime} \rho\left(\epsilon^{\prime}\right) \frac{1}{\tau_{l}(\epsilon)} .
$$

The matrix $R_{m, s}^{*}$ defining the recombination contribution from qps, one in the $m$ th and the other in the sth interval, to the total recombination rate can be written as

$$
\begin{aligned}
R_{m, s}^{*}= & \frac{1}{4 \bar{N}(0) \Delta \tau_{0}}\left(\frac{\Delta}{k_{B} T_{c}}\right)^{3} \int_{\epsilon_{m}}^{\epsilon_{m+1}} \frac{d \epsilon}{\Delta} \frac{\rho(\epsilon)}{\rho_{m}} \int_{\epsilon_{s}}^{\epsilon_{s+1}} \frac{d \epsilon^{\prime}}{\Delta} \frac{\rho\left(\epsilon^{\prime}\right)}{\rho_{s}} \\
& \times B\left(\epsilon,-\epsilon^{\prime}\right)\left[\Gamma\left(\epsilon+\epsilon^{\prime}\right) \tau_{e}\left(\epsilon+\epsilon^{\prime}\right)\right]^{-1},
\end{aligned}
$$

where $\Gamma(\epsilon)=\tau_{e}^{-1}(\epsilon)+\tau_{p h}^{-1}(\epsilon)+\tau_{p h-e}^{-1}(\epsilon)$ is the total loss rate for a phonon of energy $\epsilon$, including the effects of phonon escape from the electrode, phonon pair breaking (if $\epsilon>2 \Delta$ ) and phonon absorption by qps. It represents a generalization of the Rothwarf-Taylor recombination coefficient ${ }^{16}$ for the situation when each of colliding qps has an arbitrary energy. When both reside at a superconducting edge $(m=s=0)$, we obtain the Rothwarf-Taylor result $R_{0,0}^{*}$ $=1 /\left[4 \bar{N}(0) \Delta \tau_{0} \Gamma(2 \Delta) \tau_{e}(2 \Delta)\right]\left(2 \Delta / k_{B} T_{c}\right)^{3}$. The expressions for the elements of the matrix of tunnel rates are

$$
\Gamma_{m \rightarrow m \pm v}=\frac{1}{\rho_{m}} \int_{\epsilon_{m}}^{\epsilon_{m+1}} d \epsilon \rho(\epsilon) \Gamma_{t}(\epsilon \pm v \delta),
$$

with $\Gamma_{t}\left(\epsilon+e V_{b}\right)=\left(G / 4 e^{2} \bar{N}(0) \Omega_{0}\right) \rho\left(\epsilon+e V_{b}\right)$, where $G$ is the conductance of a barrier and $\Omega_{0}$ the electrode volume. ${ }^{33} \mathrm{Fi}$ nally, $\tau_{g, s}$, which is the rate of qp injection into the $s$ th interval due to the energy accumulation in tunneling cycles of direct and back tunneling events, ${ }^{13}$ is given by

$$
\begin{aligned}
\frac{1}{\tau_{g, s}}= & \frac{2}{\pi \tau_{0}}\left(\frac{\Delta}{k_{B} T_{c}}\right)^{3} \frac{1}{\rho_{s}} \int_{\epsilon_{m}}^{\epsilon_{m+1}} \frac{d \epsilon^{\prime}}{\Delta} \\
& \times \rho\left(\epsilon^{\prime}\right) \int_{\epsilon_{s}}^{\epsilon_{s+1}} \frac{d \epsilon^{\prime \prime}}{\Delta} \rho\left(\epsilon^{\prime \prime}\right) \int_{\Delta}^{\epsilon^{\prime \prime}-\epsilon^{\prime}-\Delta} \frac{d \epsilon^{\prime \prime \prime}}{\Delta} \\
& \times \rho\left(\epsilon^{\prime \prime \prime}\right) \rho\left(\epsilon^{\prime \prime}-\epsilon^{\prime}-\epsilon^{\prime \prime \prime}\right)\left(1-\frac{\Delta^{2}}{\epsilon^{\prime \prime} \epsilon^{\prime \prime \prime}}\right) \\
& \times \Theta\left(\epsilon^{\prime \prime}-\epsilon^{\prime}-2 \Delta\right) \frac{B\left(\epsilon^{\prime}+\epsilon^{\prime \prime \prime}-\epsilon^{\prime \prime}, \epsilon^{\prime}\right)}{\Gamma\left(\epsilon^{\prime \prime}-\epsilon^{\prime \prime \prime}\right) \tau_{p h}} .
\end{aligned}
$$

The trapping time $\tau_{m \rightarrow \text { trap }}$ describes the qp transition from a mobile state in the $m$ th interval to the trap and is assumed to be proportional to the time for the spontaneous emission of a phonon of the same energy $(m+t) \delta$. Thus,

$$
\frac{1}{\tau_{m \rightarrow \text { trap }}}=\frac{1}{\tau_{m+t \rightarrow 0}} \zeta_{\text {trap }} \text {. }
$$

The magnitude of the proportionality coefficient $\zeta_{\text {trap }}$ depends on the microscopic origin of the trap and has been introduced here as a fitting parameter. For detrapping, we write

$$
\frac{1}{\tau_{\text {detrap }}}=\sum_{s=0}^{M} \frac{1}{\tau_{\text {trap } \rightarrow s}},
$$

where the detrapping time $\tau_{\text {trap } \rightarrow \text { s }}$ refers to transitions from the trap into the $s$ th elementary interval $(s=0, \ldots, M)$ due to the absorption of a thermal phonon with energy $(s+t) \delta$. The function $K_{m, s}(t)$, describing the detrapping effect of subgap phonons, is given by

$$
\begin{aligned}
K_{m, s}(t)= & \frac{1}{\pi \tau_{p h}}\left(\frac{\Delta}{k_{B} T_{c}}\right)^{3} \frac{1}{\bar{N}(0) \Delta} \int_{(m+t) \delta}^{s \delta} d \Omega \Omega^{2} \rho\left(\Omega+\Delta_{t}\right) \\
& \times \exp \left[-\frac{t}{\tau_{e}(\Omega)}\right] \int_{\epsilon_{s}}^{\epsilon_{s+1}} \frac{d \epsilon^{\prime}}{\Delta \rho_{s}} \rho\left(\epsilon^{\prime}\right) \rho\left(\epsilon^{\prime}-\Omega\right) \\
& \times\left[1-\frac{\Delta^{2}}{\left(\epsilon^{\prime}-\Omega\right) \epsilon^{\prime}}\right],
\end{aligned}
$$

where $\tau_{e}(\Omega)$ is the loss time for subgap phonons in the STJ. ${ }^{27,28}$ Then, the array of detrapping coefficients relating to pair-breaking phonons emitted in the recombination process with participation of a trapped qp is 

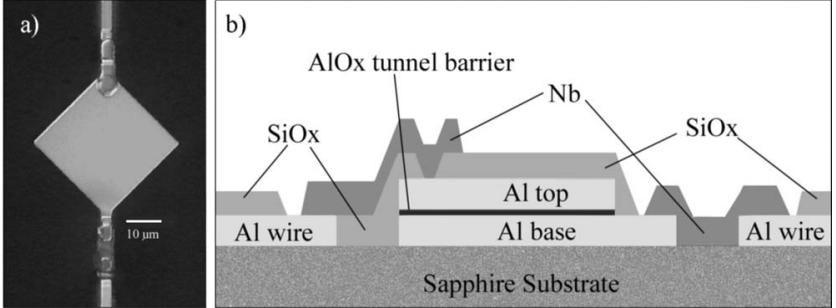

FIG. 1. (a) Micrograph of the $30 \mu \mathrm{m}$ STJ showing the Nb plugs in the leads. (b) Layer structure of the STJ. Schematic side view from the left.

$$
\begin{aligned}
R_{\text {trap }, s}= & \frac{1}{4 \bar{N}(0) \Delta \tau_{0}}\left(\frac{\Delta}{k_{B} T_{c}}\right)^{3} \int_{\epsilon_{s}}^{\epsilon_{s+1}} \frac{d \epsilon}{\Delta} \frac{\rho(\epsilon)}{\rho_{m}} \\
& \times B(\epsilon,-t \delta)\left[\Gamma(\epsilon+t \delta) \tau_{p h-e}\left(\epsilon+\epsilon^{\prime}\right)\right]^{-1} .
\end{aligned}
$$

Finally, it is convenient to split the overall qp loss rate $\tau_{l, s}{ }^{-1}$ into two distinct components, one of which, $\tau_{s \rightarrow \text { trap }}^{-1}$, arises from trapping of qps by local traps and is a strong function of qp energy, and the second, $\tau_{\text {res }}{ }^{-1}$, which describes residual losses resulting from bulk and surface recombination and outdiffusion into leads, all essentially independent of energy. Hence, the residual loss rate can be represented as a sum of the two terms, one independent of the STJ size (bulk and surface recombination) and the other inversely proportional to $L^{2}$ arising from the diffusive nature of qp transport leading to qp loss. ${ }^{30,31}$ Thus,

$$
\tau_{r e s}^{-1}=\tau_{r e s, \infty}^{-1}\left(1+\frac{a}{L^{2}}\right),
$$

where $\tau_{\text {res, },}^{-1}$ is the magnitude of the residual loss rate in the infinite sample $(L=\infty)$, that is, the residual bulk loss, and $a$ is a numerical coefficient defining the relative magnitude of outdiffusion versus bulk terms.

In the derivation of the spectral balance equations [Eq. (6)], we have ignored electron-electron interactions and the energy exchange and equilibration terms originating from self-recombination followed by sequential pair breaking. In contrast to the quadratic terms leading to recombination, such processes conserve qp number. For this reason, they are not directly relevant to recombination and detrapping and any effect arises indirectly through their modification of the qp spectral distribution. However, for nonequilibrium qp densities in typical photon absorption experiments, they are small and may be neglected.

\section{EXPERIMENT AND MODELING}

In order to demonstrate the application and success of the theoretical approach described above, we will present experimental results obtained for a series of narrow gap, multitunneling, aluminum-based STJs and discuss their analysis in terms of our model. The STJs studied were square devices, of varying sizes $L, 30,50$, and $70 \mu \mathrm{m}$ on a side, all fabricated on the same sapphire substrate with the same layer structure $100 \mathrm{~nm} \mathrm{Al} / \mathrm{AlO}_{x} / 50 \mathrm{~nm} \mathrm{Al}$ on a single chip (chip set MUL 127). An image of a typical STJ is given in Fig. 1, clearly showing the Nb plugs in the leads intended to limit qp loss through outdiffusion. Measurements were made of dc current, photoresponsivity, and charge output rise time as a function of device bias voltage and, in addition, of the dependence of responsivity and rise time on temperature and photon energy. It is important to note that the current pulse itself is not observed. The measured quantity is the total charge detected, and the time scale over which the level of $1-1 / e$ of the total charge is reached, the so-called rise time, is identically equal to the current decay time. ${ }^{32}$ Although sample measurements of $I V$ curves, responsivity, and rise time are routinely made for all STJs on all chip sets, the complete data for all measurements in the full range of variation of photon energy, bias voltage, and temperature were available only for MUL 127, obtained specifically to test the theoretical model. Experiments were carried out at temperatures between 40 and $400 \mathrm{mK}$ using either an adiabatic demagnetization cryostat $(40-200 \mathrm{mK})$ or a He sorption cooler (200-500 mK). Josephson effects were suppressed by application of a small $(\simeq 3 \mathrm{mT})$ parallel magnetic field. The apparatus was carefully shielded to ensure that no fluxoids were present in the samples. The junctions were illuminated by monochromatic near IR $(1-5 \mathrm{eV})$, multiple photon LED sources $(2-30 \mathrm{eV})$, and an ${ }^{55} \mathrm{Fe}$ radioactive source $(6 \mathrm{keV})$. Typical $I V$ characteristics are shown in Fig. 2, illustrating the effect of varying temperature and device size.

Measurements of responsivity and rise time for differentsized STJs are shown later as a function of bias voltage, photon energy, and temperature, in Figs. 3-5, respectively. For each type of measurement, the data obtained are shown
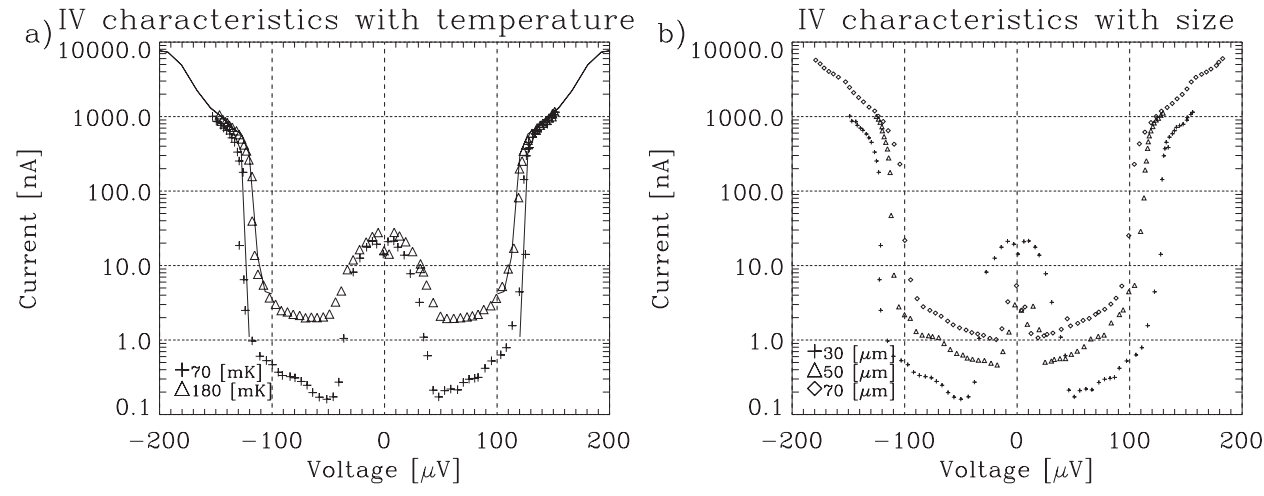

FIG. 2. $I V$ curves in Al STJ: (a) $\quad L=30 \mu \mathrm{m}, \quad T=70 \mathrm{mK}$ (crosses) and $T=180 \mathrm{mK}$ (triangles). Solid curves, theory. (b) $\quad T=40 \mathrm{mK}, \quad L=30 \mu \mathrm{m}$ (crosses), $L=50 \mu \mathrm{m}$ (triangles), and $L=70 \mu \mathrm{m}$ (diamonds). 


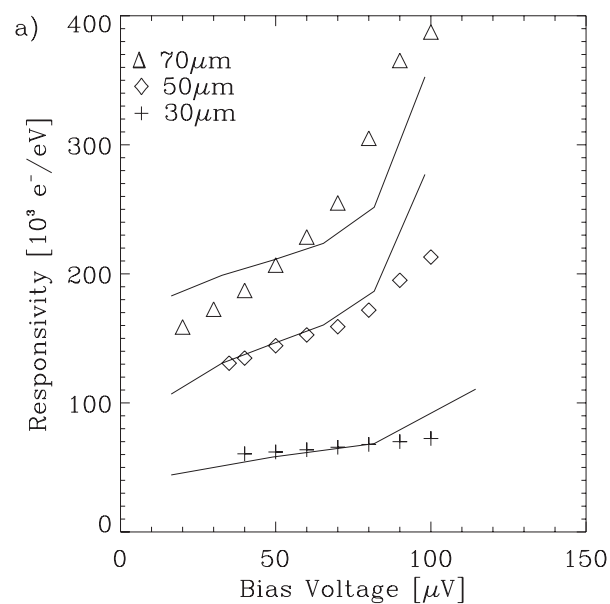

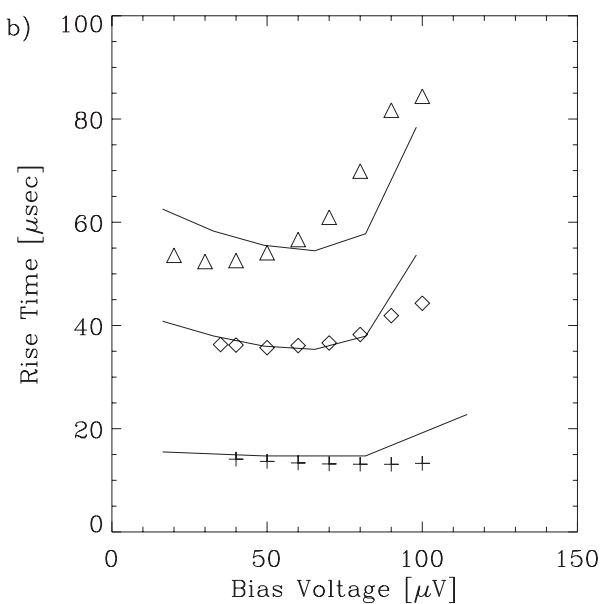

FIG. 3. (a) Responsivity and (b) rise time as a function of applied bias voltage for different device sizes at a temperature of $40 \mathrm{mK}$. as experimental points with modeled results superimposed as continuous curves. It is important to stress that the model curves for all three devices are generated using the same, single set of parameters. In contrast, within a simpler model such as that of Rothwarf and Taylor, ${ }^{16}$ a separate set of parameters, different for each STJ, would be required to model each data set, for each experiment. In addition, this model predicts monotonic decreases of both responsivity and rise time with bias voltage, which are not observed. In our model, the common parameters are of two types, first, material parameters, listed in Table I, which are obtained either from standard BCS theory or previously published in the relevant literature, or directly measured by us using standard STJ characterization procedures. For instance, the value of the effective trap depth is determined from an independent measurement of the responsivity of any of the STJs as a function of temperature. Table II given later in Sec. V contains specific parameters relating to the chip set, not previously known but obtained through the modeling procedure itself. They are $n_{t}$, the total number of traps, $\zeta_{\text {trap }}$, the trapping constant determining the residual loss time, $\tau_{\text {res }}$, the residual loss rate, and phonon escape times $\tau_{e}$ and $\tau_{e}(2 \Delta)$ for subgap and for pair-breaking phonons, respectively. The values of these fitting parameters are totally realistic on physical grounds. The total number of traps determined by the modeling is found to be the same for all STJs, implying that they reside in an area of fixed size, such as the leads to a device. The quantities that vary with device size are residual loss time, which because of the diffusive nature of qp transport contains a quadratic dependence on device size as described earlier in Eq. (18), and local trapping constant $\zeta_{\text {trap }} \propto L^{-2}$. The latter dependence is again due to the diffusive nature of qp transport delivering qps to the area where local traps reside, on the assumption that the number of local traps is independent of the STJ size. We believe that the excellent agreement between experimental results and modeled curves confirms that the behavior of such STJs is determined primarily by a strongly nonequilibrium qp distribution.

\section{ANALYSIS AND DISCUSSION OF RESULTS}

\section{A. Bias voltage dependence of dc current}

Typical dc $I V$ characteristics for the $30 \mu \mathrm{m}$ device are given in Fig. 2(a), showing the comparison between measured and calculated curves at different temperatures. In Fig. 2(b), data for all three devices, 30, 50, and $70 \mu \mathrm{m}$, are shown at the same temperature. Only the range above $\sim 100 \mu \mathrm{V}$ is meaningful; the rising current below $\sim 40 \mu \mathrm{V}$ toward zero bias is an imperfectly suppressed Josephson current, while the residual subgap current is due to leakage. We concentrate on the section of the $I V$ characteristic over which the current

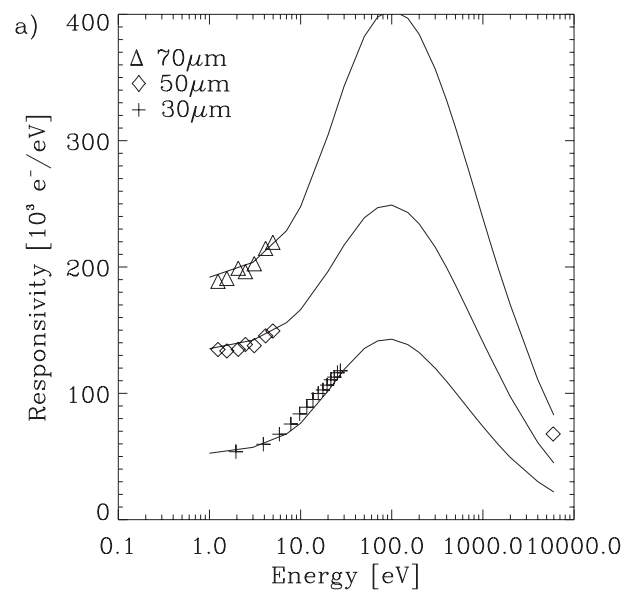

FIG. 4. (a) Measured responsivity and (b) rise time of the 30 (crosses), 50 (diamonds), and 70 (triangles) $\mu \mathrm{m} \mathrm{Al} \mathrm{junctions} \mathrm{as} \mathrm{a}$ function of incoming photon energy. $V=50 \mu \mathrm{V}, H_{\|}=5 \mathrm{mT}$, and $T=40 \mathrm{mK}$. The results of the simulations are shown by various curves. 


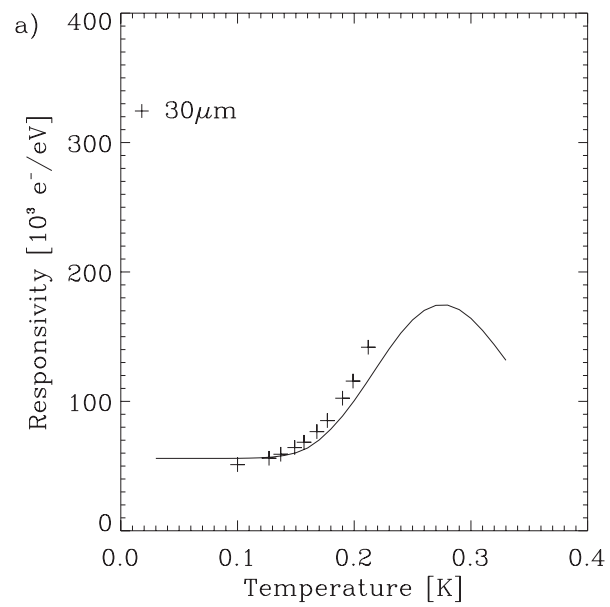

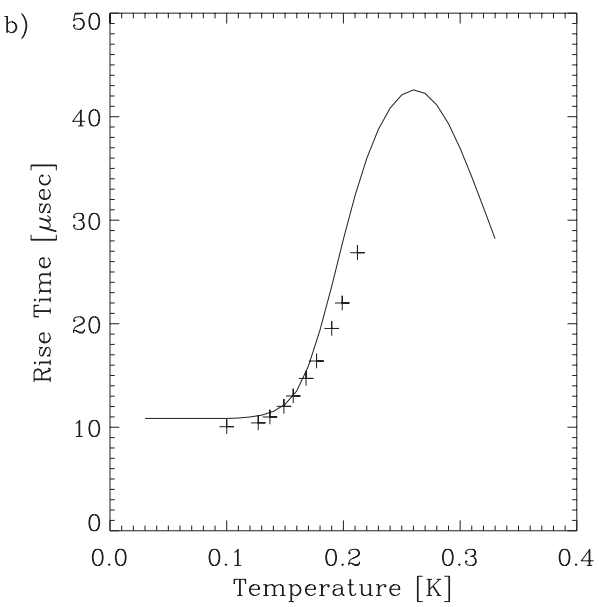

FIG. 5. (a) Responsivity and (b) rise time versus temperature. $V=85 \mu \mathrm{V}$. increases rapidly by 3-4 orders of magnitude above the relatively smooth background level at around $100 \mu \mathrm{V}$ and show that it can be attributed to the excitation of qps across the gap through multitunneling. The exact bias voltage corresponding to the current edge should be very sensitive to the qp loss rate consistent with the observation that the current edge moved toward lower bias voltage with rise in temperature [Fig. 2(a)] and increase in STJ size [Fig. 2(b)], both corresponding to lower qp loss rates.

In order to model the $I V$ characteristic, the dynamic model described in Sec. III can be simplified significantly since in the stationary situation, all time derivatives are identically equal to zero. Thus, in Eqs. (6) and (7) with $\partial / \partial t=0$, it is easy to solve the resulting system of algebraic equations to eliminate trap densities. The problem is then reduced to solving the closed system of spectral balance equations for mobile qps alone with appropriate terms to describe the interaction of qps with local traps. The latter includes detrapping due to the absorption of both thermal and nonequilibrium phonons with energy exceeding the trap depth. Spectral balance equations are obtained from Eq. (6) if $\tau_{l, s}$ is replaced by $\tau_{\text {res,s }}$ and the terms describing trapping and detrapping in Eq. (6) replaced by an effective trapping term $\tau_{s \rightarrow \text { trap }}^{\text {eff }}$ The resulting system of balance equations coincides with that of Ref. 13 but with the qp loss rate written as

$$
\frac{1}{\tau_{l, s}}=\frac{1}{\tau_{\text {res }}}+\frac{1}{\tau_{s \rightarrow \text { trap }}^{e f f}}
$$

The relation of the rise time (experimental mean loss time) $\tau$ to $\tau_{l, s}$ can only be found when it is simulated through the solution of spectral balance equations and the qp and phonon response to photon absorption calculated. The expression for $\Gamma_{s \rightarrow \text { trap }}^{\mathrm{eff}}=\left[\tau_{s \rightarrow \text { trap }}^{\mathrm{eff}}\right]^{-1}$ has the form

$$
\Gamma_{s \rightarrow \text { trap }}^{e f f}=\Gamma_{s \rightarrow \text { trap }}\left[1+\frac{\sum_{s^{\prime}} \Gamma_{s^{\prime} \rightarrow \text { trap }}^{e f f} P_{s^{\prime}}^{i}}{\Gamma_{\text {detrap }}+\sum_{s^{\prime}} R_{t s^{\prime}} P_{s^{\prime}}^{i}}\right]^{-1}
$$

where $\Gamma_{s \rightarrow \text { trap }}=1 / \tau_{s \rightarrow \text { trap }}$. Finally, the detrapping rate $\Gamma_{\text {detrap }}$ is expressed in terms of the complete distribution of phonons, including both thermal and nonequilibrium, which are capable of promoting a trapped qp into any mobile state. This rate is given by

TABLE I. STJ material characteristics used as parameters for the model.

\begin{tabular}{lcccc}
\hline \hline Symbol & Name & Value & Unit & Comment \\
\hline$R_{n}$ & Normal barrier resistivity & 6.65 & $\mu \Omega \mathrm{cm}^{2}$ & Measured \\
$\Delta$ & Energy gap & 180 & $\mu \mathrm{eV}$ & Measured \\
$\tau_{0}$ & Characteristic e-ph scattering time & 440 & $\mathrm{~ns}$ & a \\
$N(0)$ & Single spin normal state & 12.2 & $10^{23}$ & a \\
& density of states at Fermi level & & $\mathrm{eV} \mathrm{cm}^{3}$ & a \\
$\tau_{p h}$ & Characteristic pair-breaking time & 0.242 & $\mathrm{~ns}$ & Measured \\
$\Delta_{t}$ & Effective trap depth & 84 & $\mu \mathrm{eV}$ & Calculated from $R_{n}$ \\
$\Gamma_{t}(\infty)$ & Tunnel rate & 2.58 & $10^{6} / \mathrm{s}$ & \\
\hline \hline
\end{tabular}

Reference 29. 
TABLE II. Fitting parameters for the model ( $L$ in $\mu \mathrm{m})$.

\begin{tabular}{lrrr}
\hline \hline Symbol & \multicolumn{1}{c}{ Name } & Value & Unit \\
\hline$\tau_{e}(2 \Delta)$ & Escape time for pair breaking \\
\multicolumn{4}{c}{ Phonons } \\
$\tau_{e}$ & Escape time for subgap & 0.35 & $\mathrm{~ns}$ \\
\multicolumn{1}{c}{ Phonons } & 10 & $\mathrm{~ns}$ \\
$\tau_{\text {res }}^{-1}$ & Residual loss rate & $21.5 / L^{2}+0.003$ & $10^{6} \mathrm{~s}^{-1}$ \\
$\zeta_{\text {trap }}$ & Trapping constant & $72 / L^{2}$ & \\
$n_{t}$ & Number of traps & $8.8 \times 10^{3}$ & \\
\hline \hline
\end{tabular}

$$
\begin{aligned}
\Gamma_{\text {detrap }}= & \frac{1}{\tau_{0}}\left(\frac{\Delta}{k_{B} T_{c}}\right)^{3} \sum_{s^{\prime}=0} N_{t+s^{\prime}}\left(t+s^{\prime}\right)^{2}\left(\frac{\delta}{\Delta}\right)^{2} \\
& \times\left[\sqrt{\left(\frac{\epsilon_{s^{\prime}+1}}{\Delta}\right)^{2}-1}-\sqrt{\left(\frac{\epsilon_{s^{\prime}}}{\Delta}\right)^{2}-1}\right],
\end{aligned}
$$

where the expression for the phonon distribution function $N_{s}$ was obtained in the form ${ }^{13}$

$$
\begin{aligned}
N_{s}= & N_{0, s}+\frac{2 \tau_{e}(s \delta)}{\pi \tau_{p h}} \sum_{s^{\prime}} \frac{P_{s^{\prime}+s}^{i}}{\rho_{s^{\prime}+s}} \int_{\epsilon_{s}^{\prime}}^{\epsilon_{s}^{\prime}+1} \frac{d \epsilon^{\prime}}{\Delta} \rho\left(\epsilon^{\prime}\right) \rho\left(\epsilon_{s}+\epsilon^{\prime}\right) \\
& \times\left[1-\frac{\Delta^{2}}{\epsilon^{\prime}\left(\epsilon_{s}+\epsilon^{\prime}\right)}\right]
\end{aligned}
$$

where $N_{0, s}$ is the Planck distribution and subscript $s$ denotes the phonon energy of $s \delta$. After the solution of the system of spectral balance equations for qp spectral densities $P_{m}^{i}$, the current through the STJ is found as

$$
\begin{aligned}
J= & 2 e N(0) \Delta V_{S T J} \sum_{m}\left(\Gamma_{m \rightarrow m+v} P_{m}^{i}-\Gamma_{m+v \rightarrow m} P_{m+v}^{j}\right. \\
& \left.+\Theta(m-v+0)\left[\Gamma_{m \rightarrow m-v} P_{m}^{i}-\Gamma_{m-v \rightarrow m} P_{m-v}^{j}\right]\right),
\end{aligned}
$$

where $V_{S T J}$ is the STJ volume.

\section{B. Responsivity and rise time: Bias voltage dependence}

The dc, responsivity, and rise time are all calculated via a numerical solution of the spectral balance equations. However, while for dc the latter becomes a system of algebraic equations, the calculation of responsivity and rise time requires a full time-dependent solution. The simulation begins at an initial instant of time when the infinitesimally narrow initial distribution containing $N_{0}=E / 1.75 \Delta$ qps, where $E$ is the deposited energy, is taken at an arbitrary energy below $3 \Delta$, to avoid any further qp generation. The exact energy and shape of the initial distribution are of no importance ${ }^{20}$ because after a small number of tunnel events, the qp spectral distribution converges very rapidly to a stable shape, which remains unchanged during the remainder of the charge acquisition process, with only the total number of qps decreasing with time through losses. Hence, calculating the current flowing through the STJ according to Eq. (23) but with $P_{m}^{i}(t)$ as the instantaneous qp density in the $m$ th spectral interval, we may find the integrated charge $Q(t)=\int_{0}^{\infty} d t^{\prime} J\left(t^{\prime}\right)$ and determine the responsivity and rise time as

$$
\begin{gathered}
R=\left.Q(\infty)\right|_{E=1 \mathrm{eV}}, \\
Q(\tau)=\left(1-\frac{1}{e}\right) Q(\infty) .
\end{gathered}
$$

Experimental results are shown in Figs. 3(a) and 3(b). For the $30 \mu \mathrm{m}$ junction, the dependences of the responsivity and rise time on the bias voltage are rather flat. The 50 and $70 \mu \mathrm{m}$ junctions, on the other hand, show strong effects. We note that the rise time of the pulse increases with the increase of bias voltage, implying that qp losses decrease with increasing bias voltage. As a consequence, the responsivity also increases because on average qps have more time to tunnel. A second noteworthy effect is that the responsivity rises faster than the rise time, showing that not only does the lifetime of qps increase with applied voltage but at the same time tunneling becomes faster. To understand how is this possible, we need to consider the details of the quasiparticle dynamics and to examine the qp spectral distribution within the current pulse. While the bias voltage is small (well below the current edge on the $I V$ characteristic), the qp spectral distribution, although increased in breadth, still remains concentrated below the $3 \Delta$ generation threshold for all STJs. However, with increasing bias voltage, the tail of the qp spectral distribution approaches the $3 \Delta$ threshold. With all parameters except qp losses being the same for all STJs, the high energy tail of the qp distribution in larger (lower loss) STJs contains significantly more qps than in smaller devices. When the qp numbers above the $3 \Delta$ threshold become sufficiently large, self-generation occurs, resulting in a significant increase of both responsivity and rise time. As seen in Fig. 3, this occurs when the bias voltage approaches the current edge in the dc $I V$ curves and takes effect in the lowest loss $70 \mu \mathrm{m}$ STJ at the lowest bias voltages. The dc edge seen in the $30 \mu \mathrm{m}$ STJ occurs at $120 \mu \mathrm{V}$, which was beyond the range of measurements of responsivity and rise time because of the developing current instability. Examining the qp distribution functions, we calculate that in the $70 \mu \mathrm{m}$ STJ, the fraction of qps above the $3 \Delta$ threshold is of the order of $10^{-5}$ at $80 \mu \mathrm{V}$ bias. During the qp lifetime of approximately $100 \mu \mathrm{s}$, there will be on average $\sim 10^{4}$ spontaneous emissions of pair-breaking phonons resulting from qp inelastic transitions initially above the $3 \Delta$ threshold. Thus, by the time the initial distribution of qps has decayed, around $20 \%$ of it has been replaced due to self-generation, resulting in the observed behavior of responsivity and rise time.

\section{Responsivity and rise time: Photon energy dependence}

Responsivity and rise time data were measured for the 30 , 50 , and $70 \mu \mathrm{m}$ junctions as a function of photon energy between 2 and $30 \mathrm{eV}$. The results of experiment together with the modeled curves are shown in Fig. 4. For the 30 and $50 \mu \mathrm{m}$ junctions, $6 \mathrm{keV}$ data were also obtained. Nonlinearity in the optical domain arises from the fact that the number of active traps gradually saturates as the number of generated quasiparticles increases ${ }^{24}$ and from this observation, it was possible to obtain an estimate of the total number of traps. The result of approximately $8.8 \times 10^{3}$ was the same for each 
STJ regardless of size, confirming the earlier result that the traps do not reside in the bulk, nor are dispersed evenly along the device perimeter, but are concentrated at one or more well defined locations, presumably at the $\mathrm{Nb}$ contacts. In contrast, the observed responsivity of STJs with Ta contacts is essentially independent of energy over in the same range, indicating either that traps are much more numerous than in junctions with $\mathrm{Nb}$ leads or that they are absent altogether. The sensitivity of the theoretical fit to trap density and trapping coefficient is good so that this experiment may be considered as essentially a measurement of these two parameters. However, in modeling the curves in Fig. 4, theory uses also the trap depth as a parameter. The results of the simulations shown in Fig. 4 are not critically dependent on the value of this parameter, and hence the determination of $\zeta_{\text {trap }}$ and $F^{t}$ remains slightly uncertain, in the absence of an independent determination of the trap depth. The latter was achieved by measuring the STJ responsivity in the appropriate range of temperature, as described below, since thermal phonons will activate the trapped qps and thus increase responsivity with increasing temperature. ${ }^{25}$

\section{Responsivity and rise time: Temperature dependence}

Finally, we have measured the temperature dependence of responsivity and rise time in the range of temperature $40-210 \mathrm{mK}$. The results of experiments and theoretical modeling are shown in Fig. 5, from which we were able to determine the trap depth $\Delta_{t}$. In Ref. 20, detrapping rate was proposed to be proportional to that of qp absorption from the initial state at the edge, $\Delta$, into a final state above this level corresponding to the trap depth. This assumption has never been tested experimentally before and needs refinement before a quantitative modeling can be carried out. The difference between the rate of detrapping and that of phonon absorption arises from the different integrands in the expressions describing the transition rates. It is clear that absorbing a phonon of the energy exactly corresponding to the trap depth raises a trapped qp to a final state at the superconducting edge. In the BCS model, the latter is singular, leading to an enhanced detrapping rate in comparison with that proposed in Ref. 20. A realistic description of both the density of states in the vicinity of the local trap as well as of the detrapping rate requires an accurate model for the local trap. The general expression for the phonon absorption rate in an inhomogeneous superconducting system has the form ${ }^{33}$

$$
\begin{aligned}
\Gamma_{a b s}(\mathbf{x}, \epsilon)= & \frac{1}{\tau_{0}\left[k_{B} T_{c}(\mathbf{x})\right]^{3}} \int_{0}^{\Omega_{D}} d \Omega \Omega^{2} N(\Omega)[\operatorname{Re} G(\mathbf{x}, \epsilon+\Omega) \\
& \left.-\frac{\Delta(\mathbf{x})}{\epsilon} \operatorname{Im} F(\mathbf{x}, \epsilon+\Omega)\right],
\end{aligned}
$$

where $\mathbf{x}$ is a coordinate and $\operatorname{Re} G, \Delta(\mathbf{x})$, and $\operatorname{Im} F$ are position-dependent density of states, pair potential, and imaginary part of the anomalous Green function, respectively. To evaluate the detrapping rate, we take the argument in the phonon absorption rate to be $\epsilon-\Delta_{t}$. The singularity in the BCS density of states at the location of the local trap will be smoothed out because of the presence of the trap. How- ever, in spite of this singularity, the integral is convergent, and hence we expect that the difference between the two expressions for the density of states does not play a significant role. Next is the problem of estimating the pair potential $\Delta(\mathbf{x})$ at the location of the trap, taking account of the local suppression of the gap at the trap. Here, the result cannot be derived in a general form independent of the model of the local trap. If the trap is a normal region, $\Delta(\mathbf{x})$ inside the trap is zero. However, the gap itself inside this normal region, $\Delta-d_{t}$, still exists, while the pair potential is zero because $\Delta(\mathbf{x}) \sim \lambda F$, where the electron-phonon coupling constant $\lambda$ $=0$ in the normal region. Thus, we have a finite $F$ function and gap but zero $\Delta(\mathbf{x})$, a common situation in proximized structures. Of course, the result depends on our assumption about the trap region, whether it is totally normal or whether it still retains some small electron-phonon coupling. Finally, our expression for the detrapping rate is obtained from Eq. (25) by using the BCS density of states and zero pair potential at the location of the trap,

$$
\frac{1}{\tau_{\text {detrap }}}=\frac{1}{\tau_{0}\left(k_{B} T_{c}\right)^{3}} \int_{0}^{\Omega_{D}} d \Omega \Omega^{2} N(\Omega) \rho(\epsilon+\Omega) .
$$

With this expression, we may model the temperature dependence of both responsivity and rise time and compare the results with experiment to establish the value of the parameter $\Delta_{t}$. The steepness of the simulated curves on the rising side is greatly enhanced by the fact that dominant phonons excite the trapped qps into the states close to the edge where the BCS density of states is high. The results are shown in Fig. 5. In view of the several assumptions made, the agreement is promising. The general shape of the curve is similar to that observed for larger gap $\mathrm{Ta} / \mathrm{Al}$ proximized junctions ${ }^{25}$ in the region of higher temperatures, $200-800 \mathrm{mK}$, where the full curves can be measured experimentally. The responsivity and rise time curves reach maxima as a function of temperature because with rising temperature, thermal recombination first compensates for the effectively increased qp lifetimes while detrapping becomes efficient, and then completely dominates, giving rise to enhanced loss, lower responsivity, and faster rise time.

Table II lists the fitting parameters which, together with the set of material parameters of Table I, were found to model convincingly all the experimental results for all three devices. The good agreement of the model with experiment provides strong justification for the expressions used to describe loss and trapping in the STJs and therefore of the physics underlying them. The purely inverse quadratic dependence of the trapping constant, together with the observation that the number of traps is independent of STJ size, suggests strongly that the traps are localized in the $\mathrm{Nb}$ plugs at the connection with the leads. We also note that the magnitude of the bulk contribution (size independent) to the residual loss rate, corresponding to a rate of approximately $300 \mu \mathrm{s}$, is not far removed from the figure of $200 \mu \mathrm{s}$ reported in Ref. 15 for an Al STJ with Ta plugs.

\section{SUMMARY}

We have developed a theory to describe the formation and subsequent time evolution of the nonequilibrium qp state 
which is created in narrow gap, multiple tunneling STJs by the absorption of an energetic photon. The theory is based on the system of coupled dynamic equations which link qp and phonon distributions via collision integrals describing all generation, interaction, tunneling, and loss processes. No previous attempt has been successful in modeling this complex situation, which is a feature of the latest generation of high quality STJs for use at very low temperatures. For comparison, experimental measurements of responsivity and loss rate (rise time) were made on a series of Al STJs used as photon detectors. Our model was fully able to predict the responsivities and rise times and their dependence on experimental parameters such as temperature, bias voltage, and photon energy of all the related STJs in terms of a single set of material and device parameters. An important implication of the results is that local traps primarily responsible for qp loss in our Al STJs are located explicitly in the region of the $\mathrm{Nb}$ contacts. We believe that these studies provide important insight both into the physics of photoabsorption processes in STJ detectors and specifically into nonequilibrium qp phenomena in superconductors.
${ }^{1}$ E. G. Wolf, Principles of Electron Tunnelling Spectroscopy (Oxford University Press, London, 1985).

${ }^{2}$ J. C. Mather, Nature (London) 401, 654 (1999).

${ }^{3}$ C. A. Mears, S. E. Labov, M. Frank, M. A. Lindeman, L. J. Hiller, H. Netel, and A. T. Barfknecht, Nucl. Instrum. Methods Phys. Res. A 370, 53 (1996).

${ }^{4}$ P. Verhoeve, N. Rando, A. Peacock, A. van Dordrecht, and D. J. Goldie, Appl. Phys. Lett. 72, 3359 (1998).

${ }^{5}$ R. H. den Hartog, D. Martin, A. G. Kozorezov, P. Verhoeve, N. Rando, A. Peacock, G. Brammertz, M. Kramrey, D. Goldie, and R. Venn, Proc. SPIE 4012, 237 (2000).

${ }^{6}$ G. Angloher, B. Beckhoff, M. Bühler, F. v. Feilitzsch, T. Hertrich, P. Hettl, J. Höhne, M. Huber, J. Jochum, R. L. Mößbauer, J. Schnagl, F. Scholtze, and G. Ulm, Nucl. Instrum. Methods Phys. Res. A 444, 214 (2000).

${ }^{7}$ K. E. Gray, Appl. Phys. Lett. 32, 392 (1978).

${ }^{8}$ D. J. Goldie, P. L. Brink, C. Patel, N. E. Booth, and G. L. Salmon, Appl. Phys. Lett. 64, 3169 (1994).

${ }^{9}$ C. Mears, S. E. Labov, and A. Barfknecht, Appl. Phys. Lett. 63, 2961 (1993).

${ }^{10}$ P. Verhoeve, R. den Hartog, A. Kozorezov, D. Martin, A. van Dordrecht, J. K. Wigmore, and A. Peacock, J. Appl. Phys. 92, 6072 (2002).

${ }^{11}$ L. J. Hiller, M. L. van den Berg, and S. E. Labov, Appl. Phys. Lett. 79, 4441 (2001).

${ }^{12}$ A. G. Kozorezov, J. K. Wigmore, A. Peacock, R. den Hartog, D. Martin, G. Brammertz, P. Verhoeve, and N. Rando, Europhys. Lett. 66, 265 (2004).

${ }^{13}$ A. G. Kozorezov, J. K. Wigmore, A. Peacock, R. den Hartog, D. Martin, G. Brammertz, P. Verhoeve, and N. Rando, Phys. Rev. B 69, 184506 (2004).

${ }^{14}$ C. M. Wilson, L. Frunzio, and D. E. Prober, Phys. Rev. Lett. 87, 067004 (2001).

${ }^{15}$ C. M. Wilson and D. E. Prober, Phys. Rev. B 69, 094524 (2004).

${ }^{16}$ A. Rothwarf and B. N. Taylor, Phys. Rev. Lett. 19, 27 (1967).

${ }^{17}$ A. Poelaert, A. Peacock, P. Verhoeve, A. van Dordrecht, A. Owens, N. Rando, A. Kozorezov, and J. K. Wigmore, Proc.
SPIE 3445, 9214 (1998).

${ }^{18}$ K. Segall, C. Wilson, L. Frunzio, L. Li, S. Friedrich, M. C. Gaidis, and D. E. Prober, Appl. Phys. Lett. 76, 3998 (2001).

${ }^{19}$ K. Segall, C. Wilson, L. Li, L. Frunzio, S. Friedrich, M. C. Gaidis, and D. E. Prober, Phys. Rev. B 70, 214520 (2004).

${ }^{20}$ G. Brammertz, A. G. Kozorezov, J. K. Wigmore, R. den Hartog, P. Verhoeve, D. Martin, A. Peacock, A. A. Golubov, and H. Rogalla, J. Appl. Phys. 94, 5854 (2003).

${ }^{21}$ G. Brammertz, Ph.D. thesis, Universiteit Twente, 2003.

${ }^{22}$ V. F. Elesin and Yu. V. Kopaev, Sov. Phys. Usp. 24, 116 (1981).

${ }^{23}$ J. J. Chang, in Nonequilibrium Superconductivity, edited by D. N. Langenberg and A. I. Larkin (Elsevier, Amsterdam, 1986), p. 459.

${ }^{24}$ A. Poelaert, A. G. Kozorezov, A. Peacock, and J. K. Wigmore, Phys. Rev. Lett. 82, 1257 (1999).

${ }^{25}$ A. G. Kozorezov, J. K. Wigmore, A. Peacock, A. Poelaert, P. Verhoeve, R. den Hartog, and G. Brammertz, Appl. Phys. Lett. 78, 3654 (2001).

${ }^{26}$ A. Poelaert, Ph.D. thesis, Universiteit Twente, Enschede, 1999.

${ }^{27}$ G. Fagas, A. G. Kozorezov, C. J. Lambert, J. K. Wigmore, A. Peacock, A. Poelaert, and R. denHartog, Phys. Rev. B 60, 6459 (1999).

${ }^{28}$ A. G. Kozorezov, J. K. Wigmore, G. Brammertz, and A. Peacock, Phys. Status Solidi C 1, 2816 (2004).

${ }^{29}$ S. B. Kaplan, C. C. Chi, D. N. Langenberg, J. J. Chang, S. Yafarey, and D. Scalapino, Phys. Rev. B 14, 4854 (1976).

${ }^{30}$ A. G. Kozorezov, J. K. Wigmore, R. den Hartog, D. Martin, P. Verhoeve, and A. Peacock, Phys. Rev. B 66, 094510 (2002).

${ }^{31}$ R. den Hartog, A. G. Kozorezov, J. K. Wigmore, D. Martin, P. Verhoeve, A. Peacock, A. Poelaert, and G. Brammertz, Phys. Rev. B 66, 094511 (2002).

${ }^{32}$ N. Rando, A. Peacock, A. van Dordrecht, C. L. Foden, R. Engelhardt, B. G. Taylor, P. Gare, J. Lumley, and C. Pereira, Nucl. Instrum. Methods Phys. Res. A 313, 173 (1991).

${ }^{33}$ A. A. Golubov, E. P. Houwman, J. G. Gijsbertsen, J. Flokstra, H. Rogalla, J. B. leGrand, and P. A. J. deKorte, Phys. Rev. B 49, 12953 (1994). 